\title{
Hodgkin's disease in a patient with common variable immunodeficiency
}

\author{
C Christopoulos, Th Papadaki, P Vlavianos, G Kokkini
}

\begin{abstract}
A 61 year old man with long standing common variable immunodeficiency presented with pyrexia, anaemia and leucopenia. A diagnoses of Hodgkin's disease of the bone marrow was made. The typical histopathological and immunophenotypic appearances were clearly distinct from those of $T$ cell lymphoma with Reed-Sternberg-like cells which, in contrast to Hodgkin's disease, is a known complication of common variable immunodeficiency. Complete clinical and histological remission was achieved with combination chemotherapy. The latter was complicated by severe myelosuppression, unusually severe erosive mucositis and viral retinitis.

(f Clin Pathol 1995;48:871-873)
\end{abstract}

Keywords: Hodgkin's disease, common variable immunodeficiency, hypogammaglobulinaemia.

Common variable immunodeficiency (or lateonset/acquired hypogammaglobulinaemia) is a pathogenetically heterogenous entity characterised by failure of $B$ lymphocytes to produce antibody. $T$ cell dysfunction coexists in many cases and it has been suggested that the primary abnormality is failure of $\mathrm{T}$ cells to promote and regulate $B$ cell differentiation. ${ }^{12}$ There is a wide spectrum of clinical manifestations, ${ }^{134}$ including an increased incidence of malignant neoplasms, the commonest being lymphoproliferative tumours and gastric carcinoma. ${ }^{13-5}$ The lymphatic malignancies are usually nonHodgkin's lymphomas. Because of the relative rarity of the underlying disorder, little is known about the natural history and response to treatment of lymphoma in this particular setting, although there have been reports of successful use of chemo/radiotherapy. ${ }^{67}$ Here, we present a case report of Hodgkin's disease developing in a patient with common variable immunodeficiency, which highlights the unusual anatomical localisation and problems associated with the clinical management of this condition.

\section{Department of Blood} Pathology,

Evangelismos General Hospital

Athens,

Greece

Th Papadaki

Correspondence to: Dr C Christopoulos, Vas. Alexandrou 7 , Kifissia,

Athens 14561 Athens

Accepted for publication 18 January 1995 in up to $28 \%$ of patients with common immunodeficiency ${ }^{3}$ ) since the age of 30 years and mild, stable hypogammaglobulinaemia (serum $\gamma$-globulin at $75 \%$ of lower limit of normal) had been diagnosed in 1978. He had remained in good health and did not receive immunoglobulin until 1988 when he was given a single infusion of $\gamma$-globulin before a total gastrectomy for a superficial adenocarcinoma of the stomach without local lymphatic spread. He suffered no postoperative complications and remained well until early 1993 when he started experiencing frequent lower respiratory tract infections requiring treatment with multiple courses of antibiotics. In July 1993 the patient developed persistent pyrexia which did not respond to broad spectrum antibiotics.

Apart from moderate splenomegaly, physical examination was normal. The patient's haemoglobin concentration was $100 \mathrm{~g} / \mathrm{l}$, and white cell count $3.4 \times 10^{9} / 1$ (neutrophils $69 \%$, lymphocytes $17 \%$, monocytes $9 \%$, myelocytes $3 \%$, eosinophils $1 \%$, basophils $1 \%$ ) with a normal platelet count. The percentage of B lymphocytes $(\mathrm{CD} 19+)$ was within normal limits and the $T_{4} / T_{8}$ ratio was also normal at $1 \cdot 6$. The erythrocyte sedimentation rate was $44 \mathrm{~mm} /$ hour. His serum $\gamma$-globulin was $45 \%$ of the lower limit of normal: IgG $2 \cdot 8 \mathrm{~g} / 1$ (normal range 6-18), $\operatorname{IgM} 0.25 \mathrm{~g} / 1(0.5-3.5)$ and $\operatorname{IgA}$ $0 \cdot 18 \mathrm{~g} / 1(1-4 \cdot 6)$. Microbiological, serological, endoscopic, and radiological evaluation did not reveal the cause of the patient's pyrexia. Computed tomography confirmed the presence of an enlarged spleen with a vertical span of $16 \mathrm{~cm}$. A bone marrow aspirate showed iron deficiency and slightly increased numbers of megakaryocytes but was otherwise unremarkable. A trephine bone marrow biopsy specimen of the iliac crest showed extensive infiltration of the marrow by Hodgkin's disease with a diffuse pattern of involvement. The neoplastic infiltrate was polycellular with numerous ReedSternberg (RS) cells and variants, many small and medium-sized lymphocytes, benign histiocytes, few plasma cells, and neutrophils (figure, panel A). Immunophenotyping on paraffin wax sections showed that the RS cells and variants were $\mathrm{CD} 15+$ (figure, panel $\mathrm{B}$ ), $\mathrm{CD} 30+$, $\mathrm{CD} 45-, \mathrm{CD} 45 \mathrm{RO}-, \mathrm{CD} 3-$, and CD20 associated (L-26) - ; the bystander lymphocytes were predominantly of $\mathrm{T}$ cell origin (CD45 +, $\mathrm{CD} 45 \mathrm{RO}+, \mathrm{CD} 3+, \mathrm{CD} 20$ associated (L$26)$-) and only a small number were $B$ lymphocytes (CD45+, CD20 associated (L26) +, CD3-, CD45RO-). There was significant suppression of normal haemopoiesis.

Anaemia was relieved after transfusion of packed red cells through a leucocyte filter and chemotherapy was initiated with a modified EBVD (epirubicin, bleomycin, vinblastine, dacarbazine, and prednisolone) regimen. Intravenous infusions of $\gamma$-globulin $(200 \mathrm{mg} / \mathrm{kg})$ 

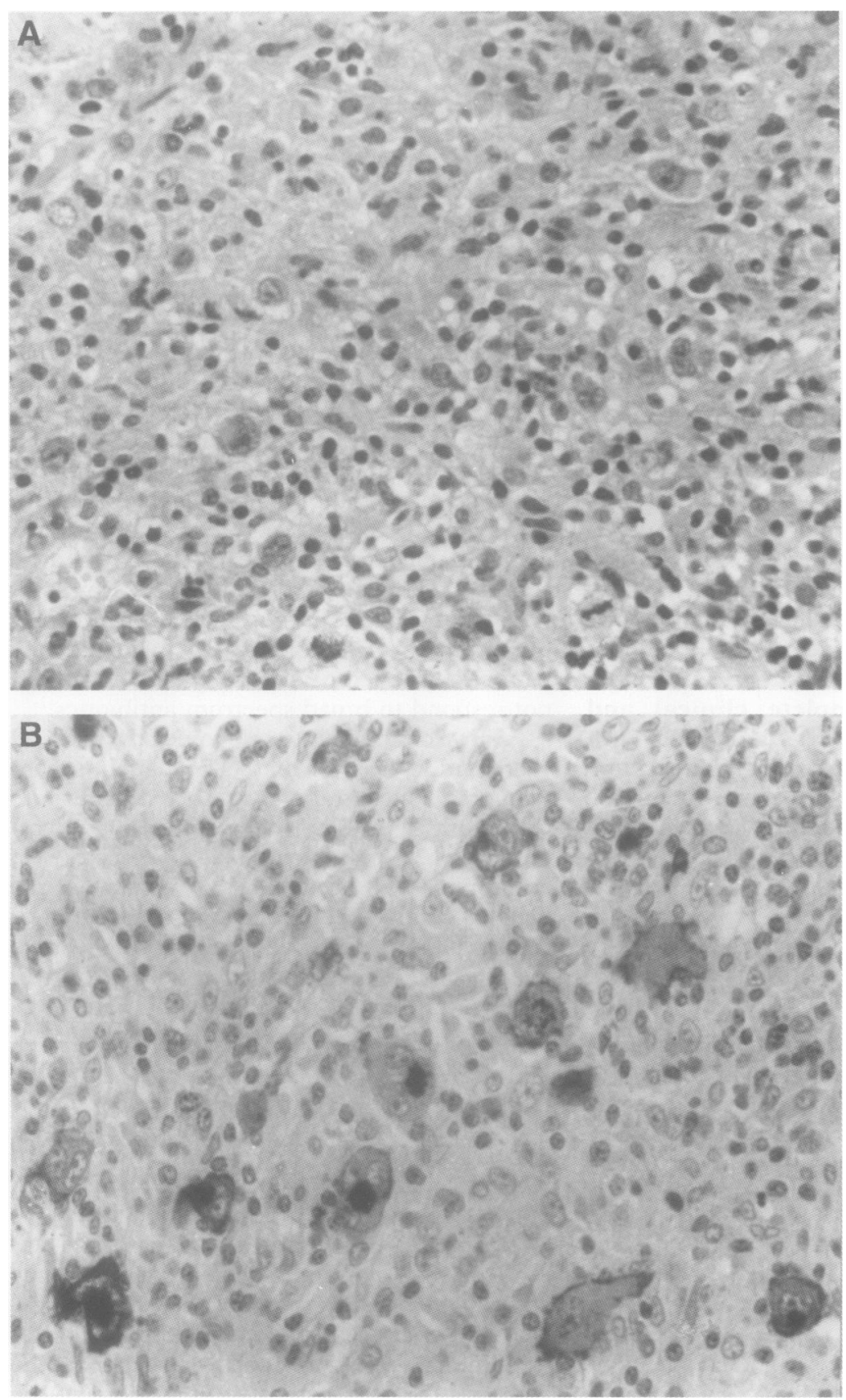

(A) A paraffin wax section of a bone marrow biopsy specimen stained with haematoxylin and eosin. RS cells and variants can be seen against a polycellular background.

(B) Paraffin wax bone marrow section stained immunohistochemically with Leu-M1 (anti-CD15) using the avidin-biotin peroxidase complex method following pretreatment in a microwave oven to enhance antigen retrieval. ${ }^{8} R S$ cells and their variants are strongly positive. which was unusually severe given the doses of cytotoxic drugs used. No pathogens were isolated at any time but pyrexia always seemed to respond to broad spectrum antibacterials. The patient was admitted to hospital with fever and abdominal pain on day 14 of the first MOPP cycle. Biopsy of an ulcerated area in the upper rectum revealed a malignant lymphocytic infiltrate with histology compatible with, but not diagnostic of, Hodgkin's disease. In the same biopsy specimen there were cellular inclusions suggestive of cytomegalovirus (CMV) infection. Polymerase chain reaction (PCR) examination of the colonic mucosa was positive for CMV. No specific antiviral treatment was given at that point in view of the absence of active colitis. Following the third MOPP cycle, the patient complained of blurred vision in the right eye and fundoscopic examination revealed florid retinitis with appearances suggestive of herpetic or CMV aetiology. PCR of aqueous humour was negative for herpes simplex, zoster, CMV, and Epstein-Barr viruses. Treatment with standard doses of intravenous foscarnet through a Hickman catheter was administered for five weeks. The frequency of the $\gamma$-globulin infusions was doubled during this time. There was a dramatic response with complete resolution of retinal changes and restoration of normal visual acuity. Foscarnet treatment was complicated by severe hypokalaemia and hypocalcaemia requiring intensive potassium and calcium replacement based on daily biochemical monitoring.

Despite the numerous treatment-related problems there was a steady improvement in the overall condition of the patient. He became apyrexial shortly after the onset of chemotherapy. Repeat sigmoidoscopy and biopsy were normal after the fourth chemotherapy cycle and his peripheral blood counts gradually returned to normal with correction of the leucopenia and anaemia. Bilateral iliac crest bone marrow biopsy specimens taken after marrow regeneration following the sixth and ninth chemotherapy cycle showed no evidence of Hodgkin's disease. A repeat computed tomography scan of the abdomen at the same time confirmed that splenomegaly had resolved. The patient is in excellent clinical condition six months after completion of chemotherapy, his only treatment consisting of monthly $\gamma$-globulin infusions $(400 \mathrm{mg} / \mathrm{kg})$.

were given every two weeks. Chemotherapy was switched to MOPP (mustine, vincristine, procarbazine, and prednisolone) following an episode of unexplained liver dysfunction which occurred after the first cycle of the EBVD regimen. The patient received three cycles of the MOPP regimen. This was followed by five cycles of HOPE-Bleo without prednisolone (doxorubicin, vincristine, bleomycin, and etoposide). Episodes of pyrexia and abdominal pain coinciding with postchemotherapy leucocyte nadirs $\left(<0.5 \times 10^{9} / 1\right.$ despite appropriate dose reductions of myelotoxic drugs and support with G-CSF) necessitated hospital admission on each of the first five chemotherapy cycles. These episodes were associated with endoscopically confirmed erosive mucositis,

\section{Discussion}

The increased incidence (varying from 1.4 to $8 \%$ ) of lymphoma in patients with common variable immunodeficiency is well documented in both prospective ${ }^{5}$ and retrospective ${ }^{134}$ studies with an apparently high frequency of undifferentiated and $T$ cell tumours. ${ }^{1}$ The diagnosis of Hodgkin's disease was made in one patient with common variable immunodeficiency in the Minnesota Immunodeficiency/Cancer Registry ${ }^{9}$ and a second case has been mentioned in the recent survey by Hermaszewski and Webster, ${ }^{4}$ although the authors comment that there was no evidence that the hypogammaglobulinaemia had preceded 
the presentation of Hodgkin's disease. There are certain points in our report that deserve comment; the first concerns the difficulties in diagnosing extranodal Hodgkin's disease. The presence of RS cells, even when they are $\mathrm{CD} 15+$, is not diagnostic of Hodgkin's disease as $\mathrm{CD} 15$ + RS-like cells have been described in T cell non-Hodgkin's lymphoma. ${ }^{10}$ RS-like cells (CD15-) were present in the bone marrow of a patient with common variable immunodeficiency who developed a peripheral $\mathrm{T}$ cell non-Hodgkin's lymphoma. ${ }^{7}$ In the case reported here, it was the combination of typical histopathology and immunophenotype that was diagnostic of Hodgkin's disease in the bone marrow. The nature of the lymphoproliferative infiltrate found on rectal biopsy was less clear but it should be noted that, although colonic localisation of Hodgkin's disease is considered extremely unusual in previously immunocompetent patients, this might not be the case in immunodeficient subjects.

Although the response of this patient to treatment was gratifying, there were many problems caused by the chemotherapy. The unusual severity of mucositis and gastrointestinal symptoms might be related to compromised mucosal defences because of his severe IgA deficiency. The rapidly advancing viral retinitis-uncommon in most immunocompetent patients undergoing chemotherapy-was compatible with the tendency of latent viruses to reactivate in patients with common variable immunodeficiency. Doubling the dose of $\gamma$ globulin during treatment with foscarnet may have played a role in the spectacular resolution of fundal changes.
In conclusion, this case report of Hodgkin's disease complicating common variable immunodeficiency illustrates that aggressive combination chemotherapy can be successful even in stage IVB disease, although unusual clinicopathological presentations and a broad spectrum of treatment related complications should be anticipated.

We thank Drs G Daikos and N Markomichelakis for help with the clinical management of this patient and Drs M Daemonacou and C Liloglou for performing histopathology studies and PCR analyses, respectively.

1 Cunningham-Rundles C. Clinical and immunologic analyses of 103 patients with common variable immunodeficiency. $f$ Clin Immunol 1989;9:22-33.

2 Spickett GP, Webster ADB, Farrant J. Cellular abnormalities in common variable immunodeficiency. Immunodefic Rev 1990;2:199-219.

3 Hermans PE, Diaz-Buxo JA, Stobo JD. Idiopathic lateonset immunoglobulin deficiency: Clinical observations in 50 patients. $\mathrm{Am} \mathcal{F} \mathrm{Med}$ 1976;61:221-37.

4 Hermaszewski RA, Webster ADB. Primary hypogammaglobulinaemia: A survey of clinical manifestations and complications. $Q \mathcal{F} \mathrm{Med}$ 1993;86:31-42.

5 Kinlen LJ, Webster ADB, Bird AG, Haile R, Peto J, Soothill $\mathrm{JF}$, et al. Prospective study of cancer in patients with $J \mathrm{~F}$, et al. Prospective study of cancer in patients

6 Gelmann E, Anderson T, Jaffe E, Broder S. Chemotherapy for lymphoma in a patient with common variable immunodeficiency. Case report, literature review and recommendations for chemotherapy in immunodeficient patients. Arch Intern Med 1982;142:90-2.

$7 \mathrm{Kim} \mathrm{JH}$, Bedrosian CL, Jain R, Schlossman DM. Peripheral T-cell lymphoma complicating common variable hypogammaglobulinaemia. Am $\mathcal{F}$ Med 1988;85:123-5.

$8 \mathrm{Shi}$ SR, Key ME, Kalva KL. Antigen retrieval in formalin fixed paraffin-embedded tissues: an enhancement method for immunohistochemical staining based on microwave oven heating of tissue sections. $\mathcal{F}$ Histochem Cytochem 1991; 39:741-8.

9 Frizzera G, Rosai J, Dehner LP, Spector BD, Kersey $\mathrm{JH}$. Lymphoreticular disorders in primary immunodeficiencies: New findings based on an up-to-date histologic classification of 35 cases. Cancer 1980;46:692-9.

10 Hyder DM, Schnitzer B. Utility of Leu M1 monoclonal antibody in the differential diagnosis of Hodgkin's disease. Arch Pathol Lab Med 1986;110:416-19.

\title{
Immunohistochemical analysis of $\mathrm{T}$ cell proliferation in normal tonsil and $B$ cell lymphoma
}

\author{
J Wang, P G Isaacson, J Spencer
}

Department of
Histopathology,
University College of
London Medical
School,
University Street,
London WC1E 6JJ
J Wang
P G Isaacson
J Spencer
Correspondence to:
Dr J Spencer.
Accepted for publication
21 February 1995

\begin{abstract}
A double immunohistochemical technique, incorporating MIB1 and CD3, was used to identify proliferating $T$ cells in paraffin wax sections of normal tonsil and $B$ cell lymphomas. The number of double stained $T$ cells as a percentage of the total $T$ cells was then determined. In normal tonsil and follicular lymphoma the follicle centre and $T$ cell zones were counted independently. In normal tonsil very few $T$ cells in the follicle centre expressed MIB1. Proliferating $T$ cells were concentrated in the $T$ cell zones. The same pattern was
\end{abstract}

observed in follicular lymphoma. In contrast, the percentage of $T$ cells expressing MIB1 was higher in mucosa associated lymphoid tissue type lymphoma and lymphocytic lymphoma, suggesting that $T$ cell activation occurs in these tumours. The highest percentage of MIB1 positive $T$ cells was observed in high grade lymphoma. This suggests that transformation to high grade lymphoma is associated with an increase in $\mathbf{T}$ cell activation.

(f Clin Pathol 1995;48:873-875)

Keywords: T cell, B cell, lymphoma, proliferation. 